André Vanzelote Barquette

\title{
Avaliação da melhor localização do sistema de mistura em linha de diesel da REDUC
}

Dissertação de Mestrado

(Opção profissional)

Dissertação apresentada como requisito parcial para obtenção do Título de Mestre (opção Profissional) pelo Programa de Pós-Graduação em Engenharia de Produção do Departamento de Engenharia Industrial da PUC-Rio.

Orientador: Prof. José Eugenio Leal

Rio de Janeiro

Março de 2008 


\section{André Vanzelote Barquette}

\section{Avaliação da melhor localização do sistema de mistura em linha de diesel da REDUC}

Dissertação apresentada como requisito parcial para obtenção do Título de Mestre (opção Profissional) pelo Programa de Pós-Graduação em Engenharia de Produção do Departamento de Engenharia Industrial da PUC-Rio. Aprovada pela Comissão Examinadora abaixo assinada.

Prof. José Eugenio Leal Orientador Departamento de Engenharia Industrial / PUC-Rio

Prof. Nélio Domingues Pizzolato Departamento de Engenharia Industrial / PUC-Rio

Álvaro Antunes Bandeira Azevedo Petróleo Brasileiro S. A.

Prof. José Eugenio Leal Coordenador Setorial de centro Técnico Científico / PUC-Rio 
Todos os direitos reservados. É proibida a reprodução total ou parcial do trabalho sem autorização da universidade, do autor e do orientador.

\section{André Vanzelote Barquette}

Graduou-se em Engenharia Química pela UFRJ em 1999. Pósgraduado em Análise, Projeto e Gerência de Sistemas pela Universidade Estácio de Sá, em 2001. Pós-graduado em Engenharia de Produção pelo Instituto Nacional de Tecnologia, em 2003. É funcionário da PETROBRAS, responsável pela Gerência Setorial de Transferência e Estocagem / Movimentação de Combustíveis da Refinaria Duque de Caxias.

Ficha Catalográfica

Barquette, André Vanzelote

Avaliação da melhor localização do sistema de mistura em linha de diesel da REDUC / André Vanzelote Barquette ; orientador: José Eugenio Leal. - 2008.

112 f. : il. ; $30 \mathrm{~cm}$

Dissertação (Mestrado em Engenharia Industrial)Pontifícia Universidade Católica do Rio de Janeiro, Rio de Janeiro, 2008.

Inclui bibliografia

1. Engenharia industrial - Teses. 2. Sistemas de mistura em linha. 3. Determinação do ponto central. 4. Logística. I. Leal, José Eugenio. II. Pontifícia Universidade Católica do Rio de Janeiro. Departamento de Engenharia Industrial. III. Título.

CDD: 658.5 


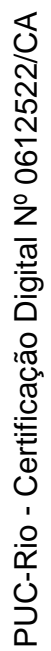

A Deus por tudo, aos meus pais, esposa e filhas pelo apoio, críticas e incentivo. 


\section{Agradecimentos}

Ao professor José Eugenio Leal, orientador da dissertação, pela oportunidade, críticas e conhecimentos transmitidos.

A Petrobras pelo patrocínio, incentivo e tempo cedido para o desenvolvimento deste trabalho.

Aos funcionários da Universidade Petrobras, pelo apoio durante todo o período do mestrado.

Aos amigos da Refinaria Duque de Caxias, pela ajuda e incentivo durante a elaboração deste trabalho.

Aos colegas do curso de mestrado profissional em logística, pelo incentivo, críticas e convívio.

Ao amigo Álvaro Antunes Bandeira Azevedo pela pronta ajuda e incentivo durante a elaboração deste trabalho.

A todos aqueles que de alguma forma me incentivaram nos momento mais difíceis para a conclusão deste trabalho. 


\section{Resumo}

Barquette, André Vanzelote. Avaliação da melhor localização do sistema de mistura em linha de diesel da REDUC. Rio de Janeiro, 2008. 112 p. Dissertação de Mestrado (Opção profissional) Departamento de Engenharia Industrial, Pontifícia Universidade Católica do Rio de Janeiro.

O objetivo da dissertação é determinar a localização ideal para a construção de um sistema de mistura em linha de diesel (MLD) na Refinaria Duque de Caxias REDUC. O modelo aplicado neste trabalho é similar ao utilizado para a determinação da melhor localização de um centro de distribuição. Neste caso, especificamente, a função objetivo do problema busca minimizar os custos com tubulações necessárias para a construção do sistema de mistura em linha numa refinaria de petróleo. Este trabalho é mais um exemplo de como a logística pode ser utilizada como ferramenta capaz de otimizar o fluxo de movimentos das origens até os destinos, visando atender com perfeição os requisitos de um complexo empreendimento de engenharia. Os resultados obtidos nesta dissertação, através da aplicação da metodologia proposta, resultaram para a Petrobras em um acréscimo significativo no retorno financeiro do projeto.

\section{Palavras-chave}

Sistemas de Mistura em Linha, Determinação do Ponto Central, Logística 


\section{Abstract}

Barquette, André Vanzelote. Assessment of better location of online blend system of diesel in REDUC. Rio de Janeiro, 2008. 112 p. MSc Dissertation - Departamento de Engenharia Industrial, Pontifícia Universidade Católica do Rio de Janeiro.

The aim of the dissertation is to determine the ideal location for the construction of an online diesel blending system in Duque de Caxias Refinery - REDUC. The model used in this study is similar to that used for determining the best location for a distribution center. In this case, specifically, the objective function of the problem is to minimize costs with pipes for the construction of the online blending system in the oil refinery. This work is another example of how the logistics can be used as a tool capable of optimizing the flow of movement of origins to the destinations, seeking perfection meet with the requirements of a complex engineering project. The results obtained in this dissertation, through the application of the methodology proposed, provided for Petrobras a significant increase in financial return of the project.

\section{Keywords}

Logistics; Determination of the central point; Online blend systems 
Índice

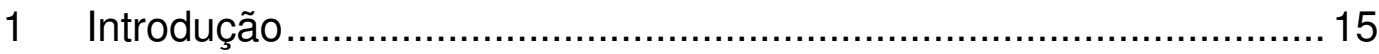

2 O Petróleo..................................................................................... 18

$2.1 \quad$ O Petróleo no Mundo ......................................................... 18

2.2 O Petróleo no Brasil .............................................................. 19

2.3 Os Constituintes do Petróleo................................................... 21

3 O Refino do Petróleo .................................................................... 24

3.1 Tipos de Processos ......................................................... 24

3.2 Processos de Separação ........................................................ 25

3.3 Processos de Conversão .................................................. 25

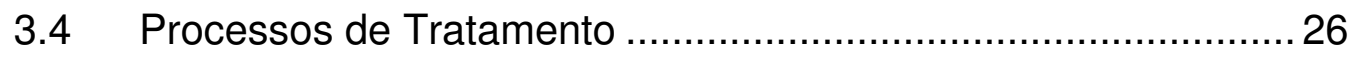

3.5 Processos Auxiliares ................................................... 26

3.6 Esquemas de Refino e as Refinarias Brasileiras ....................26

$4 \quad$ Produtos do Petróleo ................................................................ 30

4.1 Principais Derivados do Petróleo ....................................... 30

4.2 Produção de Diesel na REDUC ...................................... 32

5 O Problema de Localização dos Sistemas de Mistura em Linha ...... 35

5.1 Sistemas de Mistura em Linha ....................................... 35

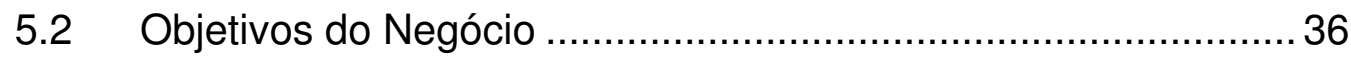

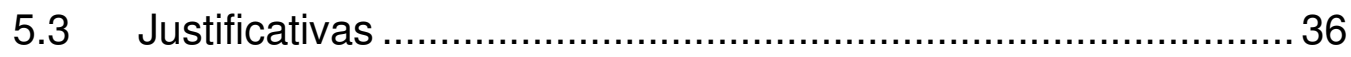

5.4 Apresentação da Tecnologia......................................... 37

5.4.1 Visão Geral .................................................................. 37

5.4.2 Controle de qualidade e otimização da mistura..................... 39

5.4.3 Analisadores em linha. ................................................. 40 
5.5.1 Localização do Sistema de Mistura em Linha para o Diesel (MLD) na REDUC 42

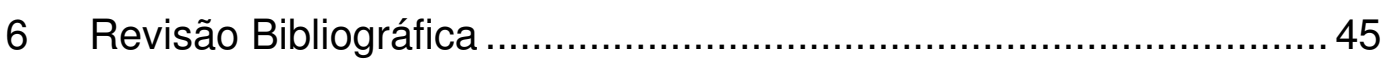

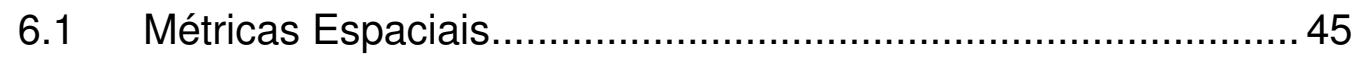

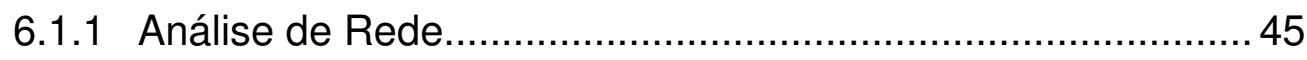

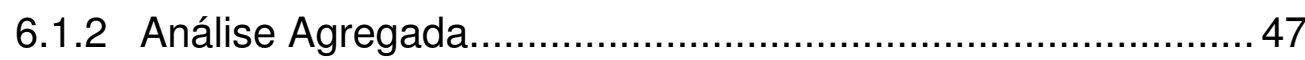

6.1.3 Análise Agregada - Métrica Retangular ....................................48

6.1.4 Análise Agregada - Métrica Euclidiana .................................... 49

6.1.5 Análise Agregada - Outras Métricas e Aproximações ............50

6.2 Sistemas de Informações Geográficas (SIG) ……....................52

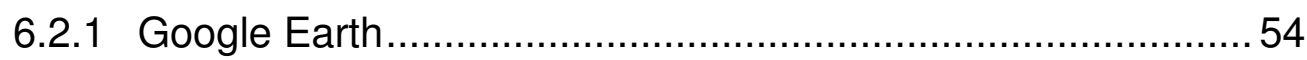

6.3 Determinação do Ponto Central .................................................55

6.3.1 Determinação do Ponto Central pela Análise em Redes Modelo das P-Medianas ............................................................. 56

6.3.2 Determinação do Ponto Central pela Análise Agregada -

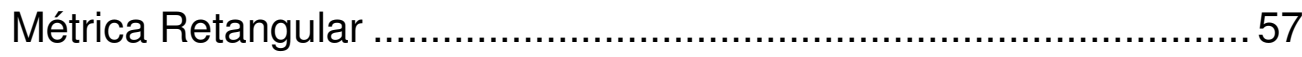

6.3.3 Determinação do Ponto Central pela Análise Agregada -

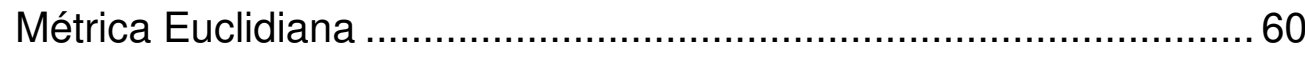

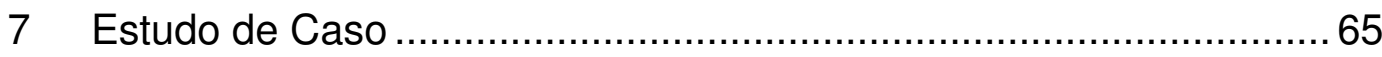

7.1 Determinação das Coordenadas X e Y de Cada Ponto ............... 65

7.2 Determinação dos Pesos entre os pontos e o ponto central......69

7.3 Determinação do Ponto Central do MLD ...................................70

7.4 Determinação do Ponto Mais Adequado para a instalação do

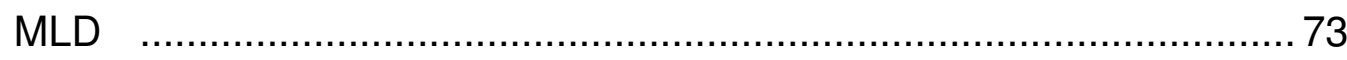

8 Síntese dos Resultados e Conclusões.............................................. 74 
8.1 Análise dos Resultados ........................................................ 74

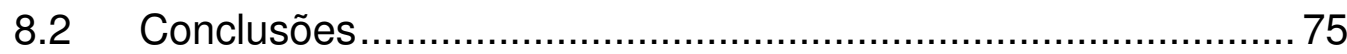

8.3 Sugestões para Novos Estudos ………….............................. 77

9 Referências Bibliográficas …........................................................ 78

ANEXO I: Os Processos de Refinação do Petróleo .................................. 81

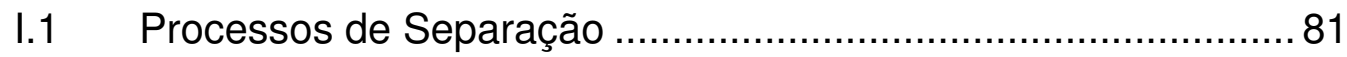

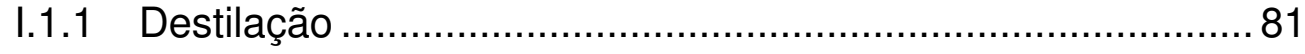

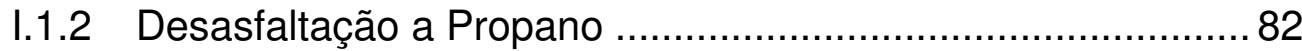

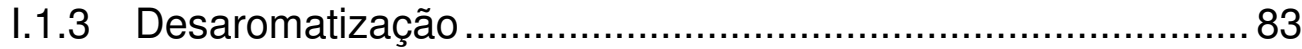

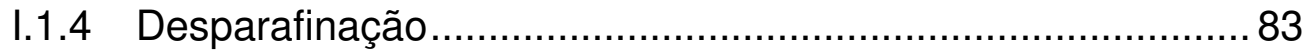

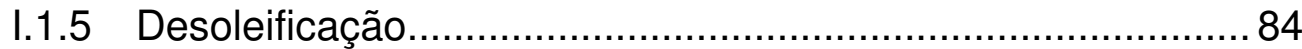

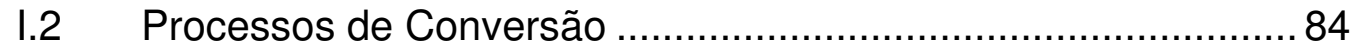

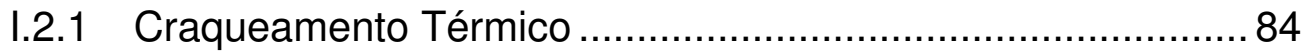

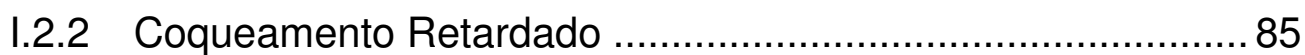

I.2.3 Craqueamento Catalítico ……………………................... 86

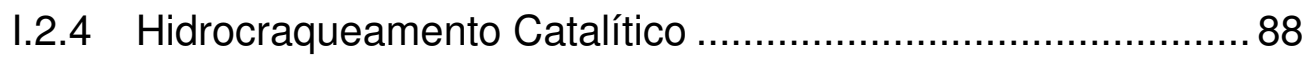

I.2.5 Hidrocraqueamento Catalítico Brando................................... 89

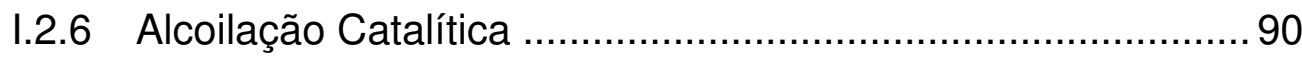

I.2.7 Reformação Catalítica ..........................................................90

I.3 Processos de Tratamento .....................................................

I.3.1 Tratamento Cáustico........................................................... 92

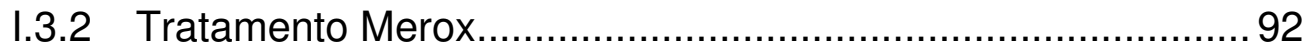

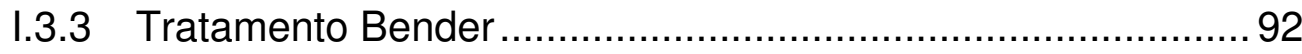

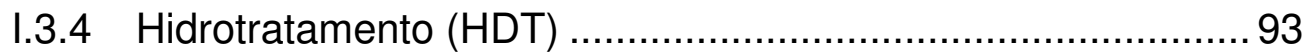

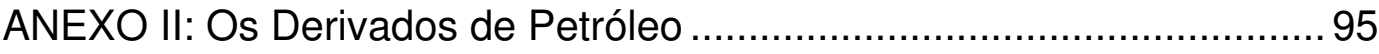




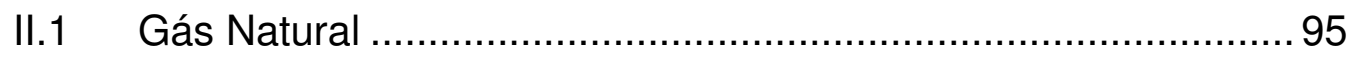

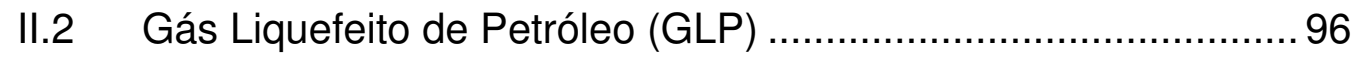

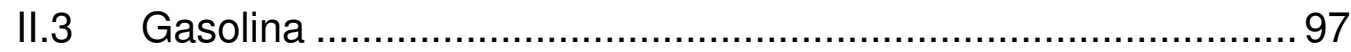

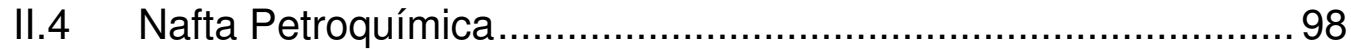

II.5 Querosene de Aviação (QAv) ………………........................ 99

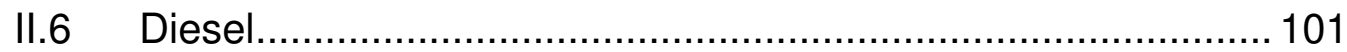

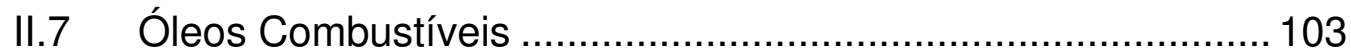

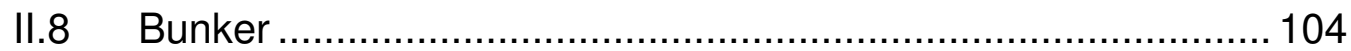

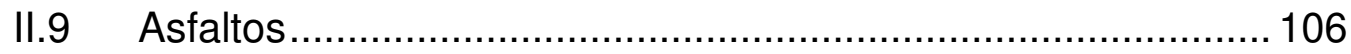

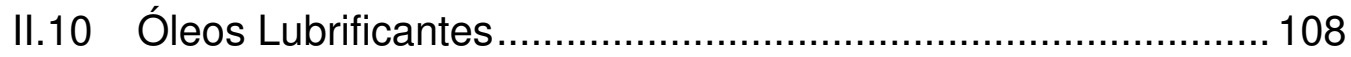

ANEXO III: Determinação do Ponto Central do MLD pelo Método Fibonaci 109

ANEXO IV: Prova do Método da Derivada ..........................................111 
Índice de Figuras

Figura 2.1: Campos de Produção de Petróleo da Bacia de Campos, RJ

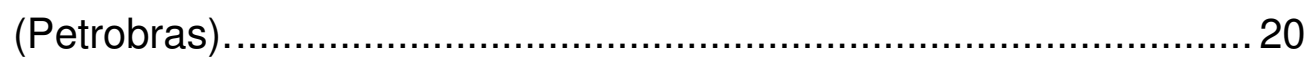

Figura 2.2: Produção de Petróleo da Bacia de Campos, RJ (Petrobras). 21

Figura 2.3: Principais Frações de Petróleo. 23

Figura 3.1: Refinarias e Fábricas de Fertilizantes da Petrobras no Brasil (Petrobras) 27

Figura 3.2: Esquema de Refino Voltado para a Produção de Combustíveis (Petrobras) 28

Figura 3.3: Esquema de Refino Voltado para a Produção de Óleos Lubrificantes (Petrobras). 29

Figura 4.1: Esquema Ideal para Maximizar a Produção de Óleo Diesel. . 33

Figura 4.2: Esquema de Produção de Óleo Diesel na REDUC 34

Figura 5.1: Estrutura de um sistema de mistura em linha genérico

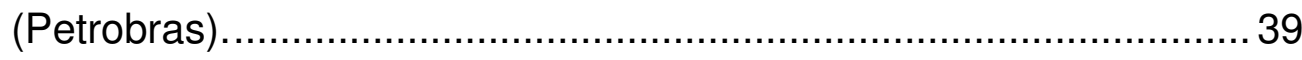

Figura 5.2: Analyzer Shelter (visão externa) - REPLAN........................... 41

Figura 5.3: Analyzer Shelter (visão interna) - REPLAN........................... 41

Figura 5.4: Analogia - Centro de distribuição e Sistemas de Mistura em Linha. 42

Figura 5.5: Alternativas de Localização - Sistema de Mistura em Linha de

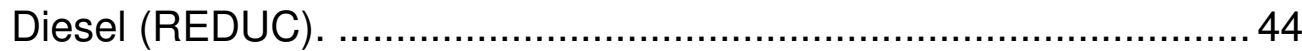

Figura 6.1: Representação de um Grafo. ............................................. 46

Figura 6.2: Métrica Retangular - Distância entre os Pontos A e B. ......... 49

Figura 6.3: Métrica Euclidiana - Distância entre os Pontos A e B. .......... 50

Figura 6.4: Google Earth - Visão Geral................................................... 54

Figura 6.5: Funcionalidades do Google Earth. ........................................ 55

Figura 7.1: REDUC Modelada Segundo Grid Cartesiano. .......................66 
Figura 7.2: Exemplo de Cálculo das Coordenas Cartesianas...................68 68

Figura I.1: Unidade de Destilação de Petróleo (Petrobras)........................81

Figura I.2: Carga e Produtos da uma Unidade de Destilação de Petróleo. 82

Figura I.3: Carga e Produtos da uma Unidade de Desasfaltação a Propano. 83

Figura 1.4: Carga e Produtos da uma Unidade de Coqueamento Retardado. 85

Figura 1.5: Unidade de Coqueamento Retardado da REDUC

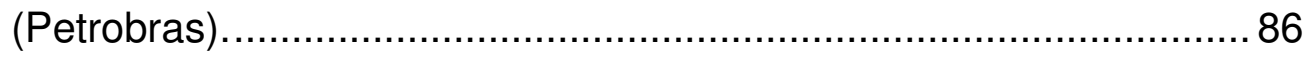

Figura I.6: Carga e Produtos da uma Unidade de Craqueamento Catalítico Fluido. 87

Figura 1.7: Unidade de Craqueamento Catalítico Fluido da REDUC

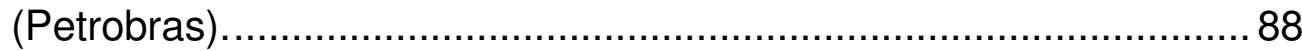

Figura I.8: Unidade de Reforma Catalítica da REDUC (Petrobras).......... 91

Figura 1.9: Unidade de Hidrotratamento de diesel da REDUC

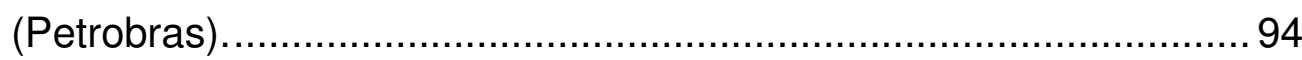

Figura III.1: Convergência pelo Método Fibonaci - Eixo X...................... 109

Figura III.2: Convergência pelo Método Fibonaci - Eixo Y..................... 110 


\section{Índice de Tabelas}

Tabela 1 - Sistemas de Mistura em linha da Petrobras ............................37

Tabela 2 - Coordenadas $X$ e $Y$ dos locais analisados ..........................6. 67

Tabela 3 - Relação Diâmetro da Tubulação X Custo por Unidade de

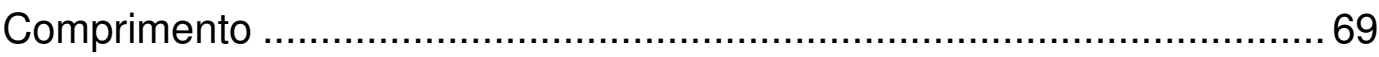

Tabela 4 - Diâmetro e Preço das Tubulações que Ligam os Pontos de Origem e Destino ao Central ........................................................... 70

Tabela 5 - Pontos Ordenados por Ordem Crescente de valor da

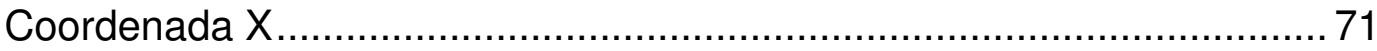

Tabela 6 - Iterações do Algoritmo para o Eixo X .................................... 71

Tabela 7 - Pontos Ordenados por Ordem Crescente de valor da

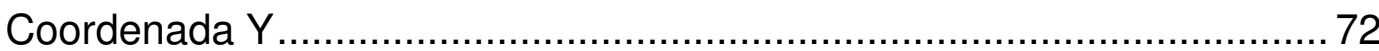

Tabela 8 - Iterações do Algoritmo para o Eixo Y ................................... 72

Tabela 9 - Pontos Passíveis de Instalação X Custo ..............................73

Tabela 10 - Metodologia X Custo ................................................... 74

Tabela 11 - Pontos Passíveis de Instalação X Acréscimo no Custo ....... 76 